\title{
Evolution: a basic science for medicine
}

\author{
Randolph M. Nesse
}

The 2009 celebrations of Darwin's birth and the publication of The Origin of Species were grand not only because his discoveries changed biology, but also because they are continuing to benefit society. Nowhere is this more evident than in medicine and public health. You might think that Darwin's discoveries would have been fully applied long ago, but a deep fracture in the intellectual landscape has prevented medicine from making full use of evolutionary biology. This is changing fast. Scientists are now recognising that diseases need evolutionary explanations as well as explanations based only on the body's mechanisms.

The field that tries to understand why natural selection has left the body vulnerable to diseases is called Darwinian medicine. Also called evolutionary medicine, it applies every aspect of evolutionary biology to every problem in medicine and public health. It has grown quickly since 1991 (Williams and Nesse, 1991). Major edited volumes have illustrated the opportunity in areas from infectious disease epidemiology to genetics, anatomy and physiology. The most significant ones are already in second editions (Stearns and Koella, 2007; Trevathan et al., 2007). They are being widely read and studied except, it seems, by physicians. Most doctors never take a course in evolutionary biology before medical school, and evolution is not part of the usual medical curriculum.

This is a significant detriment to human health. It is as if we left out embryology from the medical curriculum. Like embryology, many of the contributions of evolutionary biology are not the kind of thing that one applies in the clinic every single day. Instead, evolution provides an essential foundation for understanding why the body is the way it is, and why it fails in ways that cause disease. Physicians and researchers who understand these 
origins, and who have a deep understanding of evolutionary biology, can better prevent and treat disease.

\section{The main issues}

\section{Many applications}

Explaining the delay in fully applying evolutionary biology and medicine is a question for historians. Some are already hard at work mapping evolutionary applications in medicine from Darwin's time to present (Zampieri, 2009). In the late nineteenth and early twentieth centuries, most applications were 'medical Darwinism' that focused on the welfare of the species. In connection with eugenics, this led to moral and social disaster. Modern approaches to Darwinian medicine are entirely different. They apply evolutionary principles to improve our prevention and treatment of diseases of individuals.

Many evolutionary applications are straightforward connections to already well-established bodies of knowledge. For instance, population genetics is inherently based on evolutionary biology, and it has long been recognised that antibiotic resistance is an unfortunate example of natural selection. Studying the phylogenetic relationships among human ancestors, and among strains of pathogens, is also based on well-established methods developed by evolutionary biologists.

Doctors and public health specialists usually learn something about these techniques, but rarely do they have a chance to learn the subtleties. Sometimes advanced principles are essential to make the right decision. For instance, some well-meaning evolutionary-minded doctors have coordinated hospital policies to shift their first-choice antibiotic in concert every several months in an effort to prevent antibiotic resistance. Unfortunately, this is quite a good way to maximise the speed of developing multiple antibiotic resistance (Bergstrom et al., 2004). There is no substitute for a full understanding of evolution, with the mathematical underpinnings.

\section{Infectious disease}

While every doctor is aware of antibiotic resistance, in medical journals the concept is rarely described as 'evolution'. Instead, they avoid the 'e-word', using euphemisms such as 'arise' or 'spread'. In biology journals, it is quite the reverse; evolution is called evolution without beating around the bush (Antonovics et al., 2007). I'm not sure why medical journals avoid the word evolution, but it does not seem to be a mere accident; it accurately reflects the gulf between medical and other research into infectious disease. 
Table 6.1 Evolutionary reasons for vulnerability to disease

1. Pathogens evolve faster than hosts, and coevolution arms races shape protective defenses that can harm hosts

2. Mismatch between our bodies and the modern environment

3. Tradeoffs that have net benefits despite substantial costs

4. Constraints on what natural selection can shape

5. Selection does not shaped health and longevity, but maximal reproductive success

6. Protective responses can seem like diseases, but they are actually useful defences

Misconceptions about infection continue to be prevalent. For instance, it was long believed that extended association of a pathogen and a host led to benign coexistence. More rigorous evolutionary thinking reveals that this the case only when low virulence helps to maximise the spread of the pathogen, as for instance with viruses that cause mild colds; they spread better when people are often up and about touching other people and sneezing. For organisms spread by vectors, such as mosquitoes or the hands of a medical professional, spread tends to be faster if the pathogen makes more copies of itself faster, irrespective of what this does to the host (Ewald, 1994). Thus, malaria and cholera are often fatal. This principle has major public health implications. Sanitation procedures that prevent cholera from spreading from the sickest patients will tend to select for milder strains of cholera. The reliable use of mosquito nets will tend to decrease malaria virulence in the local population.

In a very large perspective, the evolutionary explanation for our vulnerability to infectious disease is because pathogens evolve so much faster than we do. This should provoke wonder that large, slow reproducing multicellular organisms are possible at all. It is a testimony to the effectiveness of the immune defences shaped by natural selection.

This evolutionary explanation for our vulnerability to infection, coevolution between a host and a faster-evolving pathogen, is only one of six kinds of reasons for vulnerability to disease. See Table 6.1 for a simple list of possible reasons why natural selection has not been able to further decrease our vulnerability. Every disease needs an evolutionary explanation that draws on some combination of these. Notice that more than one explanation can apply. For instance, our tendency to atherosclerosis comes from a mismatch with our modern environment, but also from tradeoffs that make a strong inflammatory response valuable in the endothelium despite its costs (Nesse and Weder, 2007).

\section{Tradeoffs}

Some scientists have the misconception that asking questions about why the body is the way it is implies an 'adaptationist' view of most aspects of the 
body as perfect. The exact opposite is the case. No aspect of the body can be perfect, because making one thing better will make something else worse. Down-regulating the immune response will decrease the risk of autoimmune disorders, but increase the risk of infection. Making bones thicker will make them less prone to breaking, but at a cost of a heavier, slower body. Such thinking about tradeoffs is a foundation for behavioural ecology and much physiology, but there are tendencies in medicine to see particular traits as all good or all bad.

Bilirubin offers a fine example. It is a breakdown product from haemoglobin. When the liver is not working properly, it accumulates in the blood, turns skin yellow, and at high concentrations causes seizures and death. Understandably, it is thought of as a toxin. However, the evolutionary question is why the body manufactures bilirubin at all. Its metabolic precursor, biliverden, is more water-soluble and thus easier to excrete. However, the body's metabolic pathways use energy to transform biliverdin into bilirubin. Then, bilirubin must return to the liver where it is conjugated with glucuronides so it can be excreted. If bilirubin were only a toxin, this would make no sense. It turns out, however, that bilirubin is a superb antioxidant. It scavenges oxygen radicals with every cycle between bilirubin and biliverdin. Modern techniques for knocking out the gene that makes the enzyme that makes this cycle possible show that without it, cells die quickly (Sedlak and Snyder, 2004). For a long-lived organism, bilirubin may be crucial to protect cells from oxidative damage.

Bilirubin levels are especially high immediately after birth. The foetal haemoglobin is broken down as it is replaced with the adult form of haemoglobin. Slightly yellow babies have been put under lights to speed conjugation and thus the excretion of bilirubin. This has made sense because bilirubin excesses can cause serious damage. Now, however, paediatricians are recognising that pre-empting the body's natural mechanisms in routine cases may be unwise, so they are somewhat increasing the levels of bilirubin that require a baby to be put under lights.

\section{Constraints}

The third reason that natural selection has not been able to make our bodies better is constraints - there are many things natural selection cannot do. Some are obvious. Replication of the DNA code cannot be perfect, so mutations creep in. One general principle, however, makes evolution very different from the process of design carried out by engineers. Engineers can start over. They can toss out the old blueprint and move the gasoline tank in an automobile to an entirely different location, or add an extra engine to an aeroplane. In contrast, natural selection can only make tiny changes, because every single version has to work. 
Our eyes would be better if they did not have a blind spot, and if the vessels and nerves did not run between the light and the retina. We, and all vertebrates, are stuck with this suboptimal system, however. The kinds of major mutations that could change it would result in blindness or something near to it, so such genetic variations would be quickly lost. It remains possible that eyes could evolve again in vertebrates; they have evolved several times independently among various organisms. However, given that our current eyes work pretty well, there would not be much selection pressure for shaping a fundamentally new visual system on top of the old one.

\section{Looking forward}

\section{Bodies are not shaped for health}

In general, individuals are healthy. If they succomb to disease and die young, their genes are less likely to be passed on to future generations. If, however, a trait results in increased reproductive success, it will tend to spread, even if it shortens lifespan. Thus, natural selection does not shape bodies mainly for health and longevity, but for reproductive success. This point comes home if you visit a nursing home. Above the age of 85, there are twice as many women as men, because mortality rates for men are higher throughout the lifespan. Exactly how high is surprising. For every 100 women who die at age 20 in most developed countries, 300 men will die. Many of the causes are from risky behaviour, but by no means all. The tradeoff between repairing tissues and competing for resources and mates gives advantages to the genes of men who invest relatively more in competitive ability than tissue repair. Of course, they do not decide to make a greater investment in competition, it is simply that their bodies have been shaped by natural selection in ways that give their genes advantages and some serious disadvantages that shorten life.

\section{Defences}

Most problems people bring to their physicians are not the direct result of a disease, but are protective responses aroused in response to something not being right. Pain, fever, cough and anxiety are useful responses shaped by natural selection in conjunction with regulation mechanisms that express them at times when they are useful. It usually seems as if these regulation mechanisms express protective responses much too easily. Otherwise, doctors should cause many more problems by using medications to block normal pain, cough, fever and anxiety. 
A simplistic approach suggests that we should simply respect the wisdom of the body and thus minimise the use of such medications. This is naïve. Doctors need to learn how natural selection shapes the mechanisms that regulate such defences so they can make good decisions for each individual patient. The foundations for such decisions are found in signal detection theory. It offers ways to calculate exactly when expressing a response is worth it. If the response is inexpensive, such as a single panic attack or a bout of coughing, or fever, and the danger is extremely expensive, such as some potential for death or serious injury, it is optimal to express the defence whenever there is even a slight chance that the danger is present (Nesse, 2005). This is called the 'smoke detector principle'.

The clinical applications are legion. For instance, it shows why the vast majority of anxiety attacks are unnecessary, even though entirely normal. One should not assume that there is something wrong in the brain of every patient who experiences a panic attack; one should instead carefully analyse the circumstances and one should make an assessment of whether the regulation mechanism is awry or not.

\section{The body is not a machine}

Enormous benefits come from established evolutionary applications to medicine, such as population genetics and methods for tracing phylogenies. Further benefits come from systematically asking why natural selection has left the body vulnerable to disease. In addition, however, an evolutionary perspective offers a more biological view of the body and its diseases. This can best be summarised by recognising that the body is not a machine shaped by engineers, it is something very different (Childs et al., 2005). The body has no blueprints, only genes that interact with environments to construct bodies that maximise reproductive success. There is no starting over with a fresh design, there are only tiny changes building atop one another in an ongoing process of bricolage that results in systems that are more complex than we would like. Some of them may be more complex than we can comprehend with our usual cognitive strategies of dividing things into neat categories with unidirectional causal arrows between them. Especially in genetics and neuroscience, new data are revealing that things are not only more complex than we had imagined, they may be indescribably complex. They can, of course, be described, just not in terms that are satisfying to human minds.

Those who do not understand how natural selection works tend to view such complexity as evidence for some other non-evolutionary explanation for organisms. They often use the phrase 'irreducible complexity'. This is deeply ironic, because extraordinary complexity is exactly what we expect from bodily systems shaped by billions of years of natural selection. There is 
nothing irreducible about it. However, if you do not understand how natural selection works, it can seem quite mysterious.

\section{Conclusions}

Many efforts are underway to provide physicians with knowledge about evolutionary biology that is comparable to the knowledge they are provided from other basic sciences (Nesse et al., 2010). The gap remains astounding and it is clear it cannot be remedied at the medical school level. New accessible books (Nesse and Williams, 1994), and textbooks (Gluckman et al., 2009) are educating a younger generation of physicians and medical researchers. They are starting courses at the undergraduate level and they will soon be making decisions about curriculum and medical schools. Nonetheless, efforts to speed this process will also speed improvements in human health. Those interested can find more information at The Evolution and Medicine Review website (http://evmedreview.com).

\section{REFERENCES}

Antonovics, J., Abbate, J.L., Baker, C.H., et al. 2007. Evolution by any other name: antibiotic resistance and avoidance of the e-word. PLoS Biology 5(2):e30.

Bergstrom, C.T., Lo, M. and Lipsitch, M. 2004. Ecological theory suggests that antimicrobial cycling will not reduce antimicrobial resistance in hospitals. Proceedings of the National Academy of Sciences USA 101:13285-90.

Childs, B., Wiener, C. and Valle, D. 2005. A science of the individual: implications for a Medical School curriculum. Annual Review of Genomics and Human Genetics 6:313-30.

Ewald, P. W. 1994. Evolution of infectious Disease. Oxford University Press, Oxford.

Gluckman, P., Beedle, A. and Hanson, M. 2009. Principles of Evolutionary Medicine. Oxford University Press, Oxford.

Nesse, R.M. 2005. Natural selection and the regulation of defenses: a signal detection analysis of the smoke detector principle. Evolution and Human Behavior 26:88-105.

Nesse, R.M. and Williams, G. C. 1994. Why We Get Sick: The New Science of Darwinian Medicine. Vintage Books, New York, NY.

Nesse, R.M. and Weder, A. 2007. Darwinian medicine: what evolutionary medicine offers to endothelium researchers. In: Aird, W. (Ed.), Endothelial Biomedicine. Cambridge University Press, Cambridge, pp. 122-8.

Nesse, R.M., Bergstrom, C.T., Ellison, P.T., et al. 2010. Making evolutionary biology a basic science for medicine. Proceedings of the National Academy of Sciences USA 107 (Suppl 1):1800-07.

Sedlak, T.W. and Snyder, S.H. 2004. Bilirubin benefits: cellular protection by a biliverdin reductase antioxidant cycle. Pediatrics 113:1776-82. 
Stearns, S.C. and Koella, J.K. (Eds). 2007. Evolution in Health and Disease. 2nd edn. Oxford University Press, Oxford.

Trevathan, W. R., McKenna, J.J. and Smith, E.O. (Eds). 2007. Evolutionary Medicine. 2nd edn. Oxford University Press, New York, NY.

Williams, G.C. and Nesse, R.M. 1991. The dawn of Darwinian medicine. Quarterly Review of Biology 66:1-22.

Zampieri, F. 2009. Medicine, evolution and natural selection: an historical overview. The Quarterly Review of Biology 84:1-23. 